\title{
Engineering of an Anti-Steroid Antibody: Amino Acid Substitutions Change Antibody Fine Specificity from Cortisol to Estradiol
}

\author{
Patrick Chames and Daniel Baty \\ Laboratoire d'Ingénierie des Systèmes Macromoléculaires, \\ Marseille, France
}

Immunoassays are widely used for determination of the concentration of steroid hormones. However, obtaining specific anti-steroid monoclonal antibodies remains difficult. We used antibody engineering and phage display methods to change the specificity of an anti-cortisol monoclonal antibody towards estradiol. This work demonstrates that production of recombinant antibodies may be a valuable way of obtaining the high-specificity antibodies required for steroid immunoassays.

Key words: Antibody; Cross-reactivity; Phage display; Single-chain variable fragment (scFv); Steroid.

\section{Introduction}

Steroid hormones have a number of different effects on their target tissues. They are involved in many cellular processes controlling metabolism, reproduction, differentiation and behaviour (1). Many clinical symptoms are associated with abnormal steroid levels. Synthetic steroids are widely used as agonists or antagonists for therapy.

Steroids are small molecules (300-400 Da) biosynthesized from cholesterol. Their molecular structures are very similar. Estradiol has a hydroxyl group at C17, whereas estrone has a ketone group. Cortisol is produced from its precursor, 11-deoxycortisol, by hydroxylation at C11. Measurement of steroid concentration is very useful during therapy and for clinical diagnosis. It is difficult to measure hormone concentrations in target cells, but blood hormone concentrations and hormone metabolites in urine can be determined. A competitive binding assay, using specific antibodies with high affinity and radioactive steroids, is the most commonly used method for measuring steroid concentrations.

However, the structural similarities of steroids often cause problems with cross-reaction in immunoassay. Plasma and urine both contain substances that crossreact with anti-steroid antibodies. The steroids can be extracted with an organic solvent and separated by chromatography before measuring their concentration by immunoassay (2-4). This method is laborious and direct immunoassay on plasma or urine extracts is preferable for multiple determinations. Unfortunately, the method used for immunoassay can significantly affect the results obtained (5). Moreover, steroids cannot themselves cause an immunological reaction and must be coupled to immunogenic proteins. The specificity of an anti-steroid antibody depends on the position of coupling to the carrier protein (6). The ring coupling position determines the cross-reactivity of the antibody (7). Cross-reactivity with different steroids may result from small but significant conformational changes in the antibody which allow it to accommodate different ligand orientations in the binding site (8). For all these reasons, no anti-estradiol antibody able to detect picogrammes of estradiol in plasma and with no cross-reaction against related steroids, has ever been produced.

Remodelling the antibody-combining site could be used to increase the specificity of an antibody. Genetic engineering techniques may make it possible to improve the binding properties of potentially useful antibodies by using mutagenesis to modify the amino acid sequence within the antibody-combining site. Substitutions in only a few amino acids can increase affinity $(9,10)$, decrease cross-reactivity with closely-related antigens (9) or change specificity (11). The use of phage display technology has made possible the genetic engineering of immunoglobulins. It is possible to mimic the immune system in vitro by expressing antibody fragments on the surface of filamentous bacteriophages (12). Antibody fragments expressed at the phage surface can be selected on the basis of specificity, affinity, avidity, or binding kinetics. Genes coding for antibody fragments can be mutated by PCR methods to increase affinity $(13,14)$, change specificity $(15)$ or modify cross-reactivity $(16,17)$. Short et al. obtained two anti-digoxin Fab mutants with affinities two and four times higher than that of the parental antibody from a phage display library containing randomized mutations of heavy chain residues (18).

X-ray crystallographic data from antibody fragments indicate that only a few residues of the antibody-combining site contribute significantly to affinity. The binding site must have some plasticity, particularly when the antigens themselves are rigid, as is the case for steroids (19). The cross-reactivity of an anti-progesterone Fab for steroid analogues may be due to flexibility of the antibody-combining site (20). Molecular modelling of the antibody-combining site may be a valuable approach for identifying residues in contact with the steroid where the X-ray structure is not known (21).

We have described the production in Escherichia coli (E. coli) of an anti-cortisol single-chain variable fragment (scFv 5A4; where the variable domains $V_{H}$ and $V_{L}$ are covalently associated by a flexible peptide) (22). The corresponding 5A4 monoclonal antibody (mAb) recognizes cortisol with a dissociation constant of $10^{-8}$ $M$ (22). Like most anti-steroid antibodies, this mAb cross-reacts with steroid analogues but does not rec- 
ognize estradiol (Fig. 1). We have also described the identification of the residues of the antibody-combining site and those which are likely to interact with cortisol (22). In the present study, we used mutagenesis to change the specificity of this anti-cortisol antibody into an anti-estradiol antibody.

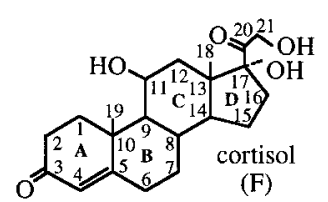

(F)

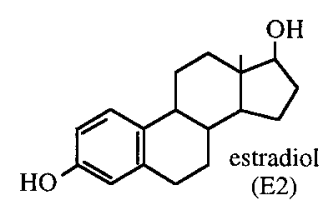

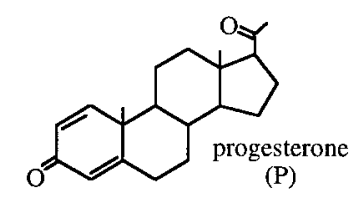

(P)

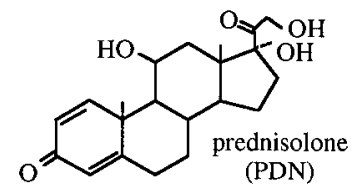

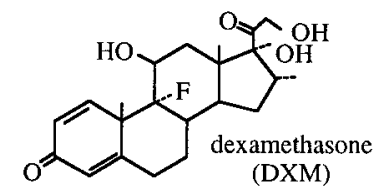

\begin{tabular}{|c|c|c|c|c|c|}
\hline F & E2 & S & P & PDN & DXM \\
\hline $100 \%$ & $<0.01 \%$ & $<0.01 \%$ & $0.08 \%$ & $33.3 \%$ & $20 \%$ \\
\hline
\end{tabular}

Fig. 1 Structures of cortisol (F), estradiol (E2), 11-deoxycortisol (S), progesterone (P), prednisolone (PDN), and dexamethasone (DXM) and their cross-reactivity with monoclonal antibody 5A4. The cross-reactivities determined by equilibrium dialysis are given as the percentage of cortisol concentration divided by steroid concentration at $50 \%$ binding of the zero standard (22).

\section{Materials and Methods}

\section{Bacterial strain}

The $E$. coli strain TG1 was used as the bacterial host for the preparation of phagemids, and as the host of bacteriophage M13K07.

\section{Construction of the wild-type anti-cortisol scFv}

To construct the $5 \mathrm{~A} 4 \mathrm{SCFV}, \mathrm{V}_{\mathrm{H}}$ and $\mathrm{V}_{\mathrm{L}}$ genes were amplified from the plasmid pscFv5A4 by PCR (22). The 3' primer used to amplify $V_{H}$ contained part of the coding sequence for the linker $\left(\mathrm{Gly}_{3} \mathrm{Ser}\right)_{4}$ and the $5^{\prime}$ primer used to amplify $\mathrm{V}_{\mathrm{L}}$ contained the rest of the sequence (with 15 overlapping bases). The scFv was assembled by splice overlap extension PCR (23). At the same time, two restriction enzyme sites ( $\mathrm{NCOl}$ and Eagl) were introduced at the $5^{\prime}$ and $3^{\prime}$ ends, respectively, of the ScFv gene to facilitate cloning into the pHENI phagemid (24) to give pHENscFv5A4.

\section{Construction of the mutant library}

We constructed the mutant $\mathrm{V}_{\mathrm{H}}$ library using recursive PCR methods (25) and a set of overlapping oligonucleotides (Fig. 2). The mutant $V_{L}$ library was constructed with a new PCRbased site-directed mutagenesis method using magnetic beads (26).

The oligonucleotides used for PCR are: oligo 1: 5' CTCGC KGCSCAGCCGGCCATGGC 3'; oligo 2: 5' CCCAGGCTTCACC AGCTCAGTTCCTGACTGCTGCAGCTGGACCTCGGCCATGGC CGGCTG 3'; oligo 3: 5' GAGCTGGTGAAGCCTGGGGCTTCA GTGAAGCTGTCCTGCAAGACTTCTGGCTTCACCTTCAGCAG TAGG 3'; oligo 4: 5' CCAGACTITTGCTTCAACCA VNNCATVN NCCTACTGCTGAAGGTG 3'; oligo 5: 5' GGTTGAAGCAAAAGT CTGGACAGAGTCTTGAGTGGATTGC 3'; oligo 6: 5' GCCTGTG

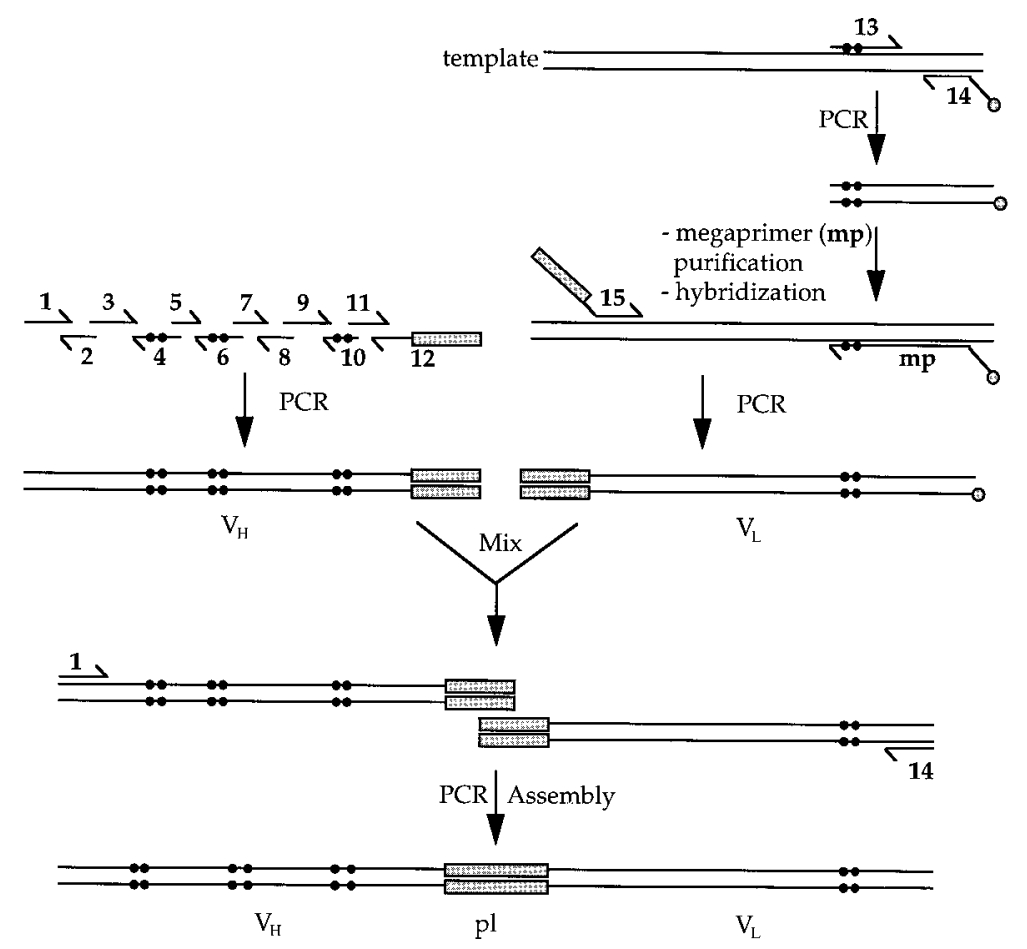

Fig. 2 PCR assembly of the scFv library. Oligonucleotides 1-12 were mixed and subjected to PCR amplification to create the mutated $V_{H}$ library. The mutated $V_{\perp}$ library was constructed using the wild-type phagemid template pHENscFv5A4 and oligonucleotides 13 and 14 for the first PCR amplification. After purification, the biotinylated single stranded megaprimer

(mp) was mixed with the template and oligonucleotide 15 for a second PCR amplification. The two mutated PCR fragment libraries $\left(V_{H}\right.$ and $\left.V_{L}\right)$ were mixed with oligonucleotides 1 and 14 and amplified by PCR to give the mutated scFv library. The dots mark the mutated positions. The boxes represent the coding sequence for the peptide linker ( $\mathrm{pl})$. 
AACTTCTGATTATA VNNAGAACCA CCAGTTCCACCA T AAATVNNTGCAATCCACTCAAGACTC 3'; oligo 7: 5' AATCA GAAGTTCACAGGCAAGGCCCAAGTGACTGTAGACACATC 3'; oligo 8: 5' GCTGAATTGCATGTAGACTGTGCTGGAGGATGTG TCTACAGTCAC 3'; oligo 9: 5' CTACATGCAATTCAGCAGCCTGACAACTGAGGACTCTGCCATCTATTACTGTGCAAG 3'; oligo 10: 5' GGCCCCAGGAATCAAAGGG VNNGCTGCTACCATAG ACACTVNNTCTTGCACAGTAATAGATGG 3'; oligo 11: 5' TT GATTCCTGGGGCCAAGGCACCTCAGTCACCGTCTCCTCA 3 ; oligo 12: 5' TCACCTGAACCAGGTITACCAGAACCTGAGGTAGAACCTGAGGAGACGGGTGAC 3'; oligo 13: 5' GCCAGCAGT GGAGTAGTNNBCCA NNBACGTTCGGTGCTGG SACNA AGBTGGA 3'; oligo 14: 5' CAAGCTTACTAGTTTATGCGGCCCCATTCAGATCC 3'; oligo 15: 5' CTGGTAAACCTGGTTCAGGT GAAGGTAGTACTAAAGGTGACATTGTGCTGAC 3'. The mutated positions are indicated by italics $(B=\mathrm{G}, \mathrm{T}$ or $\mathrm{C} ; \mathrm{K}=\mathrm{G}$ or $\mathrm{T} ; N=\mathrm{A}, \mathrm{C}, \mathrm{G}$ or $\mathrm{T} ; \mathrm{S}=\mathrm{G}$ or $\mathrm{C}$; and $V=\mathrm{G}, \mathrm{A}$ or $\mathrm{C}$ ). The codon $N N B$ was used to reduce the number of stop codons. The PCR products were purified by electrophoresis in polyacrylamide gels and the two libraries were assembled by splice overlap extension. Ncol and Eagl sites (underlined) were introduced at the same time. The final, purified PCR product $(5 \mu \mathrm{g})$ was inserted into the pHENI phagemid $(2 \mu \mathrm{g})$. Fifty electroporations were used to produce a library of $10^{8}$ clones. Some of these clones were tested by miniprep and restriction analysis for the presence of an insert of expected size. These clones were then tested for expression of a $30 \mathrm{kDa}$ product as previously described (27). The library was rescued as previously described (28) using helper phage M13KO7.

\section{Selection of phage library}

The antigen, estradiol-6-O-carboxymethyloxime-bovine serum albumin (E2-6-CMO-BSA; $10 \mu \mathrm{g} / \mathrm{ml}$ in phosphate buffered saline (PBS) solution) was incubated in maxisorp immunotubes (Nalge Nunc International, Naperville, IL, USA) overnight at $4{ }^{\circ} \mathrm{C}$. The tubes were then saturated with $2 \%$ milk PBS (MPBS) and incubated for one hour at $37{ }^{\circ} \mathrm{C}$. We used $10^{13}$ titered units of library phages in a total volume of $2 \mathrm{ml}$ MPBS per immunotube for the first round of selection. Immunotubes were washed and bound phages were eluted as previously described (28). After each round of selection, E. coli TG1 were reinfected with eluted phages and rescued to provide phages for the next round of selection. Selection was stopped when the recovery of eluted phages increased significantly $(>10$ fold, round 4 in this case).

Screening of clones using enzyme-linked immunosorbent assay

Single ampicillin-resistant colonies, resulting from infection of $E$. coliTGl with eluted phages were used to inoculate $150 \mu \mathrm{l}$ of 2 YT medium containing ampicillin $100 \mu \mathrm{g} / \mathrm{ml}$ and glucose $2 \%$, in 96 -well plates. Overnight production of phages was as previously described (28). Supernatants containing phages were tested for binding by enzyme-linked immunosorbent assay (ELISA) in Falcon 96-well plates coated with antigens (incubated at $10 \mu \mathrm{g} / \mathrm{ml}$ in PBS overnight) and saturated with MPBS. Binding of phages was detected with a horseradish peroxidase-anti-M13 antibody conjugate (Amersham Pharmacia Biotech, Uppsala, Sweden)

\section{Specificity of selected clones}

Analysis of phage binding to steroid-BSA was performed using bacterial supernatants containing phage, essentially as previously described $(29,30)$, with $10 \mu \mathrm{g} / \mathrm{ml}$ antigen in PBS in all cases, in Falcon 96-well plates.

\section{Results and Discussion}

We used the 5A4 antibody to create a library of antisteroid antibody fragments by site-directed mutagenesis. The objective was to use phage display to select from the mutants the antibody fragment with the required specificity.

We have previously cloned the scFv fragment (heavy and light variable domains linked by a flexible peptide linker) of the $5 A 4 \mathrm{mAb}$ and sequenced its gene (22). We expressed scFv fused to the g3p protein at the M13 phage surface, and used phage ELISA experiments to show that scFv had the same pattern of cross-reaction. The phage antibody immunoassay is a highly sensitive detection system. It facilitates the characterization of antibody fragments and eliminates the need for purification of antibodies (30). The 5A4 phage antibody (Phab) had very similar cross-reactivity to the parental monoclonal antibody, as is often the case (31). We have previously built a molecular model of the 5A4 paratope complexed with cortisol from the primary sequence and information about recognition of cortisol derivatives and analogues (22). This model allowed us to identify amino acids putatively in contact with the steroid. We found that the steroid nucleus may be stacked between the side chains of two tryptophans. We decided to conserve these two amino acids that could interact with any steroid, and all amino acids close to the steroid were randomized. Eight different positions were chosen in four complementarity-determining regions (CDR) (Tab. 1). The creation of a large library required large quantities of insert coding for SCFv. These mutations were mapped to sites along the entire length of the gene. Therefore, we used recursive PCR methods (25) to introduce six mutations in the $V_{H}$ gene and a new PCR-based method using magnetic beads to improve the mutagenesis efficiency in $V_{L}(26)$. Splice overlap extension (23) was then used to assemble the $\mathrm{V}_{\mathrm{H}}$ and $\mathrm{V}_{\mathrm{L}}$ library (Fig. 2). The DNA was purified, digested, ligated, and electroporated to produce a library of $10^{8}$ different clones. Library quality was tested by DNA restriction analysis and Western blot, and $96 \%$ of clones contained a full length insert and $36 \%$ of clones expressed ScFv detected by Western blotting using IPTG induction and the $9 \mathrm{E} 10$ antibody to detect the c-myc tag. Vaughan et al. obtained similar results from their library (32). The low percentage of scFv-expressing clones may be due to frame-shifts, deletions or creation of stop codons introduced during the three successive PCR steps.

Tab. 1 Residues forming the antibody-combining site in the $5 A 4$ scFv model. The mutated residues of the library are in bold script.

\begin{tabular}{ll}
\hline Chain segment & Residues \\
\hline CDR-H1 & Y33, S35 \\
FR2-H & W47 \\
CDR-H2 & W50, N58 \\
CDR-H3 & H95, T100B, F100D \\
CDR-L1 & H34 \\
CDR-L3 & W91, N94, P96 \\
\hline
\end{tabular}


The 5A4 Phab was not cross-reactive with estradiol, a steroid very different in structure to cortisol. So, after rescue using a phage helper, the library was selected against estradiol-6-CMO-BSA. Positive Phabs were detected by ELISA after the third round of selection, and almost all Phabs eluted after the fourth round were positive (Tab. 2). Sequencing of 18 positive Phabs showed that a single clone had been selected. The $\mathrm{V}_{\mathrm{H}}$ sequence of this clone (named 1B8) was identical to that of the wild-type ScFv. However, the $V_{L}$ gene had two mutations (Asn94Arg and Pro96Asp) in the CDR3. The 1B8 Phab was further characterized by ELISA (Fig. 3). Cross-reaction of the 5A4 Phab with prednisolone was not detectable with the 1B8 Phab. The 1B8 Phab gave a strong signal with estradiol but had residual binding to cortisol. Estradiol has a hydroxyl group at position C3. This position is close to the two mutations in the mutant Phab in our model. These new amino acids may form a hydrogen bond at this position, allowing recognition of estradiol. However, only part of the original paratope was modified and this may explain the residual binding to cortisol. It is highly improbable that selection led to conservation of wild-type codons in the $V_{H}$ gene. The selected wild-type $V_{H}$ gene may result from contamination during the construction of the mutant library. It may also indicate that the gene coding for the $\mathrm{V}_{\mathrm{H}}$ domain in the original library may have been mutated too much, destabilizing the paratope scaffold. This problem could be overcome by using the parsimonious mutagenesis method that limits the number of amino acids that do not retain parental structural features (33). The mutation rate is controlled and can be limited to two or three mutations per clone over the eight degenerated positions.

Tab. 2 Frequency of binding clones from the scFv-phage library. The number of phage-transfecting units (T. U.) is given before (input) and after (output) each round of selection. Forty eight clones in each round of selection were tested by ELISA for their capacity to bind estradiol-6-CMO-BSA.

\begin{tabular}{lllll}
\hline & \multicolumn{4}{l}{ Round of selection } \\
\cline { 2 - 5 } & 1 & 2 & 3 & 4 \\
\hline Input phages (T.U.) & $10^{13}$ & $5.10^{12}$ & $5.10^{12}$ & $5.10^{12}$ \\
Output phages (T.U.) & $10^{5}$ & $10^{5}$ & $5.10^{5}$ & $5.10^{8}$ \\
Binders & $0 / 48$ & $0 / 48$ & $4 / 48$ & $46 / 48$ \\
\hline
\end{tabular}

We selected a new anti-estradiol antibody fragment by molecular modelling, site-directed randomization and phage display. The specificity of this clone could be improved. For example, chain-shuffling (34) could be used to select a $V_{H}$ partner that specifically interacts with another site of the steroid. The resulting clone may have higher affinity for estradiol and may lose its affinity for cortisol. This work demonstrates that antibody engineering is a valuable technique for obtaining specific anti-steroid antibodies for immunoassays.

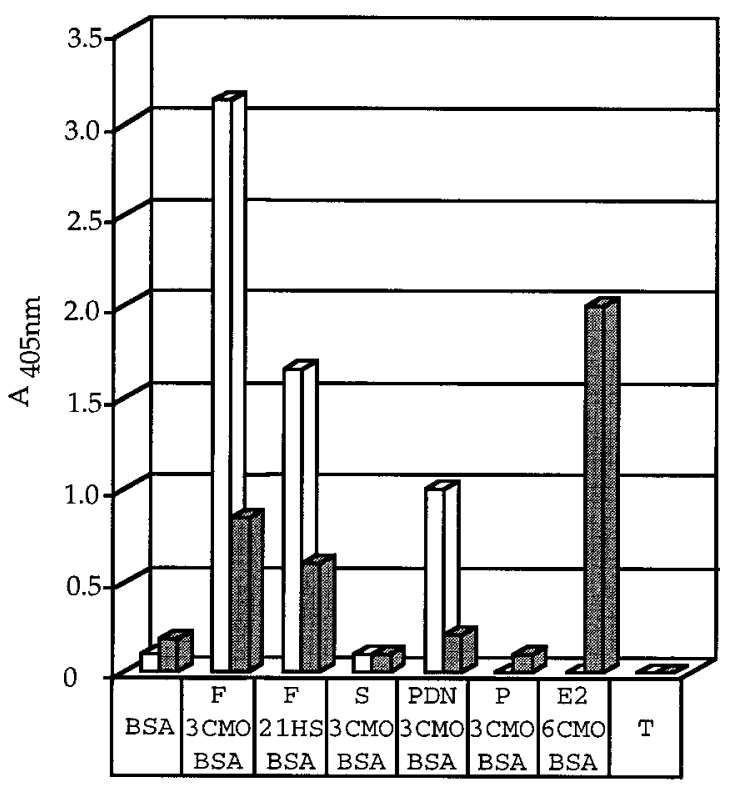

Fig. 3 Specificity of 5A4 and 1B8 Phabs. ELISA was used to test Phabs for their ability to bind directly to steroid-BSA. Cortisol-3-O-carboxymethyloxime-BSA (F-3-CMO-BSA), cortisol-21hemisuccinate-BSA (F-21-HS-BSA), 11-deoxycortisol-3-CMOBSA (S), prednisolone-3-CMO-BSA (PDN), progesterone-3CMO-BSA (P), estradiol-6-CMO-BSA (E2), BSA or milk (T) were used to coat $96-$ well plates. Culture supernatants containing Phabs were incubated with the plates for one hour at room temperature. The plates were washed and bound Phabs were detected by addition of horseradish peroxidase/anti-M 13 conjugate. The absorbance, indicated by bars, was measured at $405 \mathrm{~nm}$. $\square$ 5A4 Phab, $\square$ 1B8 Phab

\section{Acknowledgements}

We thank C. Y. Cuilleron and E. Mappus for the gift of steroidBSA and helpful discussion. We are very grateful to D. Duché and J. Chauveau for critical reading and helpful discussion and to M. Chartier for technical assistance. This work was supported by the Centre National de la Recherche Scientifique.

\section{References}

1. Milgrom E. Steroid hormones. In: Baulieu EE, Kelly PA, editors. Hormones: from molecules to disease. Paris: Hermann, 1990:385-437.

2. Barkley MS, Lasley BL, Thompson MA, Shackleton $\mathrm{CH}$. Equol: a contributor to enigmatic immunoassay measurements of estrogen. Steroids 1985; 46:587-608.

3. Agasan AL, Stewart BJ , Watson TG. Development of a radioimmunoassay method for ethynylestradiol in plasma using a monoclonal antibody. J Immunol Methods 1994; 177:251-60.

4. Wudt SA, Wachter UA, Homoki J , Teller WM. 17 alphahydroxyprogesterone, 4-androstenedione, and testosterone profiled by routine stable isotope dilution/gas chromatography-mass spectrometry in plasma of children. Pediatr Res 1995; 38:76-80.

5. Cook NJ , Read GF. Oestradiol measurement in women on oral hormone replacement therapy: the validity of commercial test kits. Br J Biomed Sci 1995; 52:97-101.

6. Yoon DY, Choi MJ , Choe IS, Chung TW, Byun SM. Influence of the conjugation site on the specificity of monoclonal 
antibodies to progesterone and on the performance of direct enzyme immunoassay. Biochem Mol Biol Int 1993; 31:553-63.

7. Gani M, Coley J , Piron J , Humphreys AS, Arevalo J , Wilson $I A$, et al. Monoclonal antibodies against progesterone: effect of steroid-carrier coupling position on antibody specificity. J Steroid Biochem Mol Biol 1994; 48:277-82.

8. Arevalo J H, Hassig CA, Stura EA, Sims MJ, Taussig MJ , Wilson IA. Structural analysis specificity. Detailed comparison of five Fab'-steroid complexes. J Mol Biol 1994; 241:663-90.

9. Roberts S, Cheetham J C, Rees AR. Generation of an antibody with enhanced affinity and specificity for its antigen by protein engineering. Nature 1987; 328:731-4.

10. Sharon J . Structural correlates of high antibody affinity: three engineered amino acid substitutions can increase the affinity of anti-p-azophenylarsonate antibody 200-fold. Proc Natl Acad Sci USA 1990; 87:4814-7.

11. Kussie PH, Parhami-Seren B, Wysocki LJ , Margolies MN. A single engineered amino acid substitution changes antibody fine specificity J Immunol 1994; 152:146-52.

12. Marks J D, Hoogenboom HR, Griffiths AD, Winter G. Molecular evolution of proteins on filamentous phage: mimicking the strategy of the immune system. J Biol Chem 1992; 267:16007-10.

13. Deng SJ, MacKenzie CR, Sodowska J, Michniewicz J, Young NM, Bundle DR, et al. Selection of antibody singlechain variable fragments with improved carbohydrate binding by phage display. J Biol Chem 1994; 269:9533-8.

14. Schier R, McCall A, Adams GP, Marshall KW, Merritt H, Yim $M$, et al. Isolation of picomolar affinity anti-c-erbB-2 singlechain Fv by molecular evolution of the complementary determining regions in the center of the antibody binding site. J Mol Biol 1996; 263:551-67.

15. Casson LP, Manser T. Evaluation of loss and change of specificity resulting from random mutagenesis of antibody VH region. J Immunol 1995; 155:5647-54.

16. Glaser SM, Yelton DE, Huse WD. Antibody engineering by codon-based mutagenesis in a filamentous phage vector system. J Immunol 1992; 149:3903-13.

17. Barbas CF, Hu D, Dunlop N, Sawyer L, Cababa D, Hendry $\mathrm{RM}$, et al. In vitro evolution of a neutralizing human antibody to human immunodeficiency virus type 1 to enhance affinity and broaden strain cross-reactivity. Proc Natl Acad Sci USA 1994; 91:3809-13.

18. Short MK, J effrey PD, Kwong RF, Margolies MN. Contribution of antibody heavy chain CDR1 to digoxin binding analyzed by random mutagenesis of phage-displayed Fab 2610. J Biol Chem 1995; 270:28541-50.

19. Arevalo J H, Stura, EA, Taussig MJ , Wilson IA. Three-dimensional structure of an anti-steroid Fab' and progesterone-Fab' complex.J Mol Biol 1993; 231:103-18.

20. Arevalo J H, Taussig MJ , Wilson IA. Molecular basis of the crossreactivity and the limits of antibody-antigen complementarity. Nature 1993; 365:859-63.

21. J ackson T, Morris BA, Martin ACR, Lewis DVF, Sanders PG. Molecular modelling and site-directed mutagenesis on a bovine anti-testosterone monoclonal antibody. Protein Eng 1992; 5:343-50.

22. Le Calvez H, Fieschi J , Green J M, Marchesi N, Chauveau J , Baty D. Paratope characterization by structural modelling of two anti-cortisol single-chain variable fragments produced in E. coli. Mol Immunol 1995; 32:185-98.

23. Horton RM, Hunt HD, Ho SN, Pullen J K, Pease LR. Engineering hybrid genes without the use of restriction enzymes: gene splicing by overlap extension. Gene 1989; 77:61-8.

24. Hoogenboom HR, Griffiths AD, J ohnson KS, Chiswell DJ , Hudson P, Winter G. Multi-subunit proteins on the surface of filamentous phage: methodologies for displaying antibody (Fab) heavy and light chains. Nucleic Acids Res 1991; 19:4133-7.

25. Prodromou C, Pearl LH. Recursive PCR: a novel technique for total gene synthesis. Protein Eng 1992; 5:827-9.

26. Fieschi J , Niccoli P, Camilla C, Chames P, Chartier M, Baty D. Polymerase chain reaction-based site-directed mutagenesis using magnetic beads. Anal Biochem 1996; 234:210-4.

27. Harrison J L, Williams SC, Winter G, Nissim A. Screening of phage antibody libraries. Methods Enzymol 1996; 267:83-109.

28. Marks J D, Hoogenboom HR, Bonnert TP, McCafferty J , Griffiths AD, Winter G. By-passing immunization: human antibodies from V-gene libraries displayed on phage. J Mol Biol 1991; 222:581-97.

29. Hoogenboom HR, Winter G. By-passing immunisation: human antibodies from synthetic repertoires of germline $\mathrm{VH}$ gene segments rearranged in vitro. J Mol Biol 1992; 227:381-8.

30. Li Y, Owen MR, Cockburn W, Kumagai I, Whitelam GC. Study of antibody-antigen interaction through site-directed mutagenesis of the $\mathrm{VH}$ region of a hybrid phageantibody fragment. Protein Eng 1996; 9:1211-7.

31. Skerra A. Bacterial expression of immunoglobulin fragments. Curr Opin Immunol 1993; 5:256-2.

32. Vaughan TJ , Williams AJ , Pritchard K, Osbourn J K, Pope $A R$, Earnshaw J C, et al. Human antibodies with sub-nanomolar affinities isolated from a large non-immunized phage display library. Nature Biotechnol 1996; 14:309-14.

33. Schier R, Balint RF, McCall A, Apell G, Larrick J W, Marks $J$ D. Identification of functional and structural amino acid residues by parsimonious mutagenesis. Gene 1996; 169:147-55.

34. Marks J D, Griffiths AD, Malmqvist M, Clackson TP, Bye J M, Winter G. By-passing immunization building affinity human antibodies by chain shuffling. Biotechnology 1992; 10:779-83.

Received 5 November 1997; accepted 17 April 1998

Corresponding author: Dr. D. Baty, UPR 9027, CNRS, IBSM, 31 chemin J oseph Aiguier, F-13402 Marseille Cedex 20, France Tel.: +334911641 17, Fax: +33491712124

E-mail: baty@ibsm.cnrs-mrs.fr 